\title{
Crescimento físico e estado nutricional de crianças e adolescentes da região de Cotinguiba, Sergipe
}

\author{
Physical growth and nutritional status of children and adolescents from Cotinguiba, Northeast Brazil
}

\section{Edio Luiz Petroski ${ }^{1}$ Roberto Jerônimo S. Silva², Andreia Pelegrini ${ }^{3}$}

\section{RESUMO}

Objetivo: Investigar o crescimento físico e o estado nutricional de crianças e adolescentes da região de Cotinguiba, Sergipe.

Métodos: Trata-se de um estudo transversal, realizado com 1.257 escolares de sete a 14 anos. Mediram-se o peso corporal e a estatura. O estado nutricional foi definido conforme as recomendações propostas pela International Obesity Task Force, de acordo com o índice de massa corpórea (IMC), segundo idade e sexo. Foram classificados com desnutrição os escolares com IMC entre 16 e $18,5 \mathrm{~kg} / \mathrm{m}^{2}$ e com excesso de peso aqueles com IMC igual ou superior a $25 \mathrm{~kg} / \mathrm{m}^{2}$.

Resultados: Um suposto pico de crescimento em estatura foi verificado aos 11 e 12 anos para os sexos feminino e masculino, respectivamente. Para o peso corporal, o pico ocorreu aos 11 anos de idade, para ambos os sexos. As curvas do National Center for Health Statistics (NCHS) foram estatisticamente superiores em todas as idades e em ambos os sexos para peso corporal e estatura, com exceção da idade de nove anos para o sexo feminino. Verificou-se que $21,7 \%$ dos escolares apresentaram desnutrição (18,5\% em meninos e 24,6\% em meninas) e $6,8 \%$ apresentaram excesso de peso $(5,1 \%$ em meninos e $8,4 \%$ em meninas), com diferenças significativas entre os sexos.

Conclusões: $\mathrm{O}$ crescimento das crianças e adolescentes da região de Cotinguiba não acompanha as curvas de referência do NCHS. A prevalência elevada de desnutrição evidencia a necessidade de um acompanhamento longitudinal do crescimento desta população e da implementação de estratégias de intervenção para reduzir tais proporções, que indicam se tratar de um problema de Saúde Pública.

Palavras-chave: antropometria; crescimento; estado nutricional; índice de massa corporal.

'Doutor em Educação Física pela Universidade Federal de Santa Maria, professor do Programa de Pós-graduação em Educação Física da Universidade Federal de Santa Catarina (UFSC) e líder do Grupo de Pesquisa em Cineantropometria e Desempenho Humano ( $\mathrm{NuClDH}$ ). Florianópolis, SC, Brasil

${ }^{2}$ Mestre em Educação Física pela UFSC e professor da Universidade Tiradentes. Aracajú, SE, Brasil

${ }^{3}$ Mestranda em Educação Física do Programa de Pós-graduação em Educação Física da UFSC e membro do Grupo de Pesquisa NuCIDH. Florianópolis, SC, Brasil

\section{ABSTRACT}

Objective: To study the physical growth and the nutritional status of children and adolescents from the region of Cotinguiba, Sergipe, in Northeast Brazil.

Methods: This cross-sectional study enrolled 1,257 schoolchildren aged seven to 14 years old. Body weight and height were measured in all studied population. Nutritional status was defined by the International Obesity Task Force recommendations, according to body mass index (BMI), by age and sex. Malnutrition was classified as a BMI between 16 and $18.5 \mathrm{~kg} / \mathrm{m}^{2}$ and overweight as a BMI greater than or equal to $25 \mathrm{~kg} / \mathrm{m}^{2}$.

Results: An apparent peak of height growth was observed in girls with 11 years old and in boys with 12 years old. Peak weight gain was noted at 11 years for both genders. At all ages and in both genders, the body weight and the height of the studied children and adolescents were below National Center for Health Statistics (NCHS) values, with the exception of girls who were nine years old. The overall prevalence of malnutrition was $21.7 \%$ (18.5\% for boys and $24.6 \%$ for girls) and the prevalence of overweight was $6.8 \%$ (5.1\% for boys and $8.4 \%$ for girls), with significant differences between genders.

Conclusions: The growth of children and adolescents in the region of Cotinguiba (Northeast Brazil) does not fit in the NCHS reference curves. The high prevalence of malnutrition indicates the need for further studies regarding the growth of this population and the need for implementing intervention strategies to reduce the problem. Malnutrition is still a public health problem in this region.

Key-words: anthropometry; growth; nutritional status; body mass index.
Endereço para correspondência:

Edio Luis Petroski

NuCIDH - Centros de Desportos - UFSC

Caixa Postal 476 - Campus Universitário - Trindade

CEP 88040-900 - Florianópolis/SC

E-mail: petroski@cds.ufsc.br

Fonte financiadora: bolsa de estudo pela Coordenação de Aperfeiçoamento de Pessoal de Nível Superior (Capes)

Recebido em: 24/1/2008

Aprovado em: 1/6/2008 


\section{Introdução}

O crescimento físico caracteriza-se pela somatória de fenômenos celulares, biológicos, bioquímicos e morfológicos, cuja interação é efetuada por meio de um plano pré-determinado geneticamente e influenciada pelo meio ambiente ${ }^{(1)}$. Em termos populacionais, os padrões de crescimento têm diversas aplicações, tais como: detectar a situação atual do indivíduo quanto aos padrões nutricionais, avaliar as práticas de introdução da alimentação complementar, além de rastrear e acompanhar grupos de risco nutricional ${ }^{(2)}$.

O controle do crescimento e do estado nutricional em crianças e adolescentes tem sido caracterizado como um importante componente da saúde materna e infantil. Portanto, as pesquisas de caráter transversal permitem determinar a magnitude de um possível problema nutricional em diferentes grupos populacionais ${ }^{(3,4)}$. Desta forma, para monitorar o crescimento físico, na área clínica e de saúde pública, são utilizadas tradicionalmente medidas antropométricas de peso corporal e estatura, as quais apresentam aceitação internacional para detectar a qualidade social, econômica e política do ambiente ${ }^{(5)}$.

Estudos sobre crescimento físico em crianças e adolescentes têm sido realizados em vários países; dentre eles, destaca-se aquele desenvolvido pelo National Center for Health Statistics $(\mathrm{NCHS})^{(6)}$ com crianças norte-americanas, cuja finalidade foi propor referenciais normativos voltados à monitoração do crescimento físico. Recentemente, o referencial de crescimento do NCHS, utilizado mundialmente desde 1977, foi revisado, com o objetivo de corrigir algumas falhas, e vem sendo referido como curvas de crescimento do Centers for Disease Control (CDC), 2000 ${ }^{(7)}$. Em nosso meio, o Ministério da Saúde adota as informações produzidas pelo NCHS como referências normativas para monitorar o crescimento físico da população ${ }^{(8)}$.

No Brasil, dois estudos relevantes e de grande projeção nacional tentaram construir um padrão nacional de crescimento. Um deles, realizado em Santo André, São Paulo ${ }^{(9)}$, verificou que crianças pertencentes às famílias de alta renda mostravam crescimento semelhante ao encontrado pelo NCHS. O outro estudo, promovido pelo Instituto Nacional de Alimentação e Nutrição (INAN), desenvolveu uma curva de crescimento da população brasileira de zero a $25 \operatorname{anos}^{(10)}$. Além desses estudos, iniciativas isoladas também avaliaram diferentes regiões do Brasil ${ }^{(4,5,11,12)}$.

Nos países em desenvolvimento, como o Brasil, existem diferenças no padrão de vida entre as regiões e, até mesmo, dentro da mesma região. Assim, estudar os níveis de saúde de uma determinada população, no sentido de enfatizar a prevenção primária e a promoção da saúde, deve ser uma prioridade em Saúde Pública. O presente estudo objetivou investigar o crescimento físico e o estado nutricional de crianças e adolescentes da região de Cotinguiba, Sergipe.

\section{Métodos}

A análise do crescimento físico e do estado nutricional de crianças e adolescentes da região de Cotinguiba foi desenvolvida a partir de um estudo epidemiológico transversal, em uma amostra representativa dos escolares do ensino fundamental da região.

Antes da coleta dos dados, houve contato com a direção das escolas e esclarecimentos sobre os procedimentos a serem realizados e os objetivos do estudo. Os pais ou responsáveis autorizaram a participação dos filhos por meio da assinatura de um formulário de consentimento livre e esclarecido. Os protocolos de intervenção no estudo foram aprovados pelo Comitê de Ética em Pesquisa da Universidade Federal de Santa Catarina (UFSC).

O estado de Sergipe está dividido em 75 municípios e cinco regiões geoeconômicas: Litoral, Cotinguiba, Agreste, Baixo São Francisco e Sertão. A região de Cotinguiba é composta por 13 municípios, tendo como principal atividade econômica a produção de cana-de-açúcar. De acordo com o Censo 2000, estavam matriculados regularmente 25.379 escolares, sendo $5 \%$ (1.267) na rede de ensino particular, $58,1 \%$ (14.751) na municipal e 36,9\% (9.361) na estadual. O processo amostral foi selecionado em dois estágios, com unidade amostral por município (Japaratuba, Capela, Maruim, General Maynard, Muribeca, Rosário do Catete e Carmópolis) e série escolar (primeira à oitava série). Nos municípios com mais de uma escola, foram selecionadas aquelas com o maior número de alunos na faixa etária em estudo. Desta forma, dez escolas estaduais participaram da pesquisa.

Para determinar o tamanho amostral, recorreu-se à fórmula proposta por Barbeta ${ }^{(13)}$ e, para efeito de cálculo, considerou-se uma prevalência de obesidade de $10 \%$, com intervalo de confiança de $95 \%$ (IC95\%) e erro estimado de 5\%. Desta forma, a amostra foi constituída de 1.257 escolares de ambos os sexos, da rede estadual de ensino, com idade de sete a 14 anos e divididos em dois grupos etários: crianças (sete a nove anos) e adolescentes (dez a 14 anos) ${ }^{(14)}$.

Foram incluídos no estudo os escolares aparentemente saudáveis e livres de tratamento médico. Consideraram-se como perda amostral os escolares cujos pais não autorizaram 
a participação e aqueles que se ausentaram da escola no dia específico da coleta de dados.

Medidas antropométricas de peso corporal e estatura foram mensuradas segundo procedimentos padronizados ${ }^{(15)}$. Os indicadores nutricionais foram definidos conforme as recomendações propostas pela International Obesity Task Force (IOTF) ${ }^{(16,17)}$, segundo idade e sexo. Assim, a desnutrição foi definida para os escolares com índice de massa corpórea (IMC) entre 16 e $18,5 \mathrm{~kg} / \mathrm{m}^{2(17)}$ e o excesso de peso $^{(16)}$ para aqueles com IMC igual ou superior a $25 \mathrm{~kg} /$ $\mathrm{m}^{2}$. A expressão "excesso de peso" foi adotada para definir sobrepeso e obesidade.

Para comparar as variáveis de crescimento físico por idade cronológica, de acordo com o sexo, foi utilizada a análise de variância. O teste $t$ de Student foi empregado para comparar as variáveis de crescimento físico do presente estudo com as curvas de referência (NCHS) por sexo e idade. A prevalência de desnutrição e excesso de peso foi comparada por meio do teste do qui-quadrado, segundo sexo e faixa etária. As diferenças entre sexo e faixa etária foram analisadas por meio do teste de comparação para duas proporções. Em todas as análises, adotou-se nível de significância de $5 \%$.

\section{Resultados}

Dos 1.257 escolares (480 crianças e 777 adolescentes) investigados no presente estudo, $53 \%$ (667) eram femininos e $47 \%$ (590) masculinos. Com relação aos valores descritivos da estatura, peso corporal e IMC, foram observados aumentos gradativos para todas as idades em ambos os sexos. Houve diferenças significativas entre os sexos apenas nas idades de nove e 14 anos, sendo as escolares do sexo feminino mais altas aos nove anos e os do sexo masculino mais altos aos 14 anos $(p<0,05)$. O IMC das meninas foi estatisticamente superior ao dos meninos na idade de 14 anos $(p<0,05)$ (Tabela 1 ).

A Tabela 2 demonstra um suposto pico de crescimento médio de $7,63 \mathrm{~cm} /$ ano em estatura nas meninas entre 11 e 12 anos e, nos meninos, o pico foi de 7,86 cm/ano entre 12 e 13 anos. Notou-se, ainda, um aumento médio significativo do crescimento em todas as faixas etárias, com exceção dos intervalos etários de 9-10 e 13-14 anos para o sexo feminino e 10-11 anos para o masculino. Em relação ao peso corporal, médias mais elevadas foram encontradas entre 11 e 12 anos para ambos os sexos. Nas meninas houve um acréscimo de $6,1 \mathrm{~kg} / \mathrm{ano}$, com diferenças significativas

Tabela 1 - Valores descritivos para estatura, peso corporal e índice de massa corpórea de acordo com sexo e idade

\begin{tabular}{lcccccc}
\hline \multirow{2}{*}{ Idade (anos) } & \multicolumn{2}{c}{ Estatura $(\mathbf{c m})$} & \multicolumn{2}{c}{ Peso corporal $\mathbf{( k g )}$} & \multicolumn{2}{c}{ IMC $\left.\mathbf{( k g} \mathbf{m}^{\mathbf{2}}\right)$} \\
\cline { 2 - 6 } & Feminino & Masculino & Feminino & Masculino & Feminino & Masculino \\
\hline 7 & $120,11 \pm 5,48$ & $121,78 \pm 6,46$ & $22,14 \pm 4,30$ & $22,24 \pm 3,85$ & $15,19 \pm 2,04$ & $14,97 \pm 1,95$ \\
8 & $126,08 \pm 6,55$ & $126,14 \pm 6,73$ & $24,35 \pm 4,44$ & $24,44 \pm 4,43$ & $15,24 \pm 1,91$ & $15,26 \pm 1,67$ \\
9 & $132,80 \pm 7,95$ & $130,22 \pm 6,61^{*}$ & $28,31 \pm 5,81$ & $26,79 \pm 5,25$ & $15,92 \pm 2,01$ & $15,69 \pm 1,93$ \\
10 & $136,15 \pm 6,72$ & $136,37 \pm 7,14$ & $30,42 \pm 7,22$ & $30,97 \pm 6,94$ & $16,26 \pm 2,80$ & $16,51 \pm 2,54$ \\
11 & $141,03 \pm 6,98$ & $139,68 \pm 6,47$ & $33,71 \pm 6,45$ & $32,20 \pm 5,28$ & $16,89 \pm 2,71$ & $16,41 \pm 1,71$ \\
12 & $148,66 \pm 7,47$ & $146,62 \pm 7,61$ & $39,59 \pm 9,51$ & $38,37 \pm 10,10$ & $17,77 \pm 3,35$ & $17,70 \pm 3,64$ \\
13 & $153,81 \pm 6,90$ & $154,48 \pm 9,50$ & $43,28 \pm 9,39$ & $43,66 \pm 9,91$ & $18,16 \pm 3,09$ & $18,17 \pm 3,30$ \\
14 & $155,48 \pm 6,15$ & $158,37 \pm 8,76^{*}$ & $47,19 \pm 8,42$ & $46,17 \pm 9,46$ & $19,46 \pm 2,92$ & $18,30 \pm 2,73^{*}$ \\
\hline
\end{tabular}

${ }^{*} p<0,05$ entre os sexos nas comparações em sujeitos da mesma idade.

Tabela 2 - Valores médios do crescimento anual em crianças e adolescentes, segundo sexo e grupos etários

\begin{tabular}{|c|c|c|c|c|c|c|}
\hline \multirow{2}{*}{ Intervalo etário } & \multicolumn{3}{|c|}{ Feminino } & \multicolumn{3}{|c|}{ Masculino } \\
\hline & Estatura & Peso corporal & IMC & Estatura & Peso corporal & IMC \\
\hline 7 7-8 & $5,91^{*}$ & 2,23 & 0,02 & $4,33^{*}$ & 0,88 & $-0,61$ \\
\hline 8-19 & $6,72^{*}$ & $3,96^{*}$ & 0,68 & $4,08^{*}$ & 2,35 & 0,43 \\
\hline $9-10$ & 3,35 & 2,11 & 0,34 & $6,15^{*}$ & 4,18 & 0,82 \\
\hline $10-11$ & $4,88^{*}$ & 3,29 & 0,63 & 3,31 & 1,23 & $-0,10$ \\
\hline $11 \dashv 12$ & $7,63^{*}$ & $5,88^{*}$ & 0,88 & $6,94^{*}$ & $6,17^{*}$ & $1,29 *$ \\
\hline $13-14$ & 1,67 & $3,91^{*}$ & $1,30^{*}$ & $3,89^{*}$ & 2,51 & 0,13 \\
\hline
\end{tabular}

${ }^{*} p \leq 0,05$ entre os intervalos etários para cada variável analisada. 


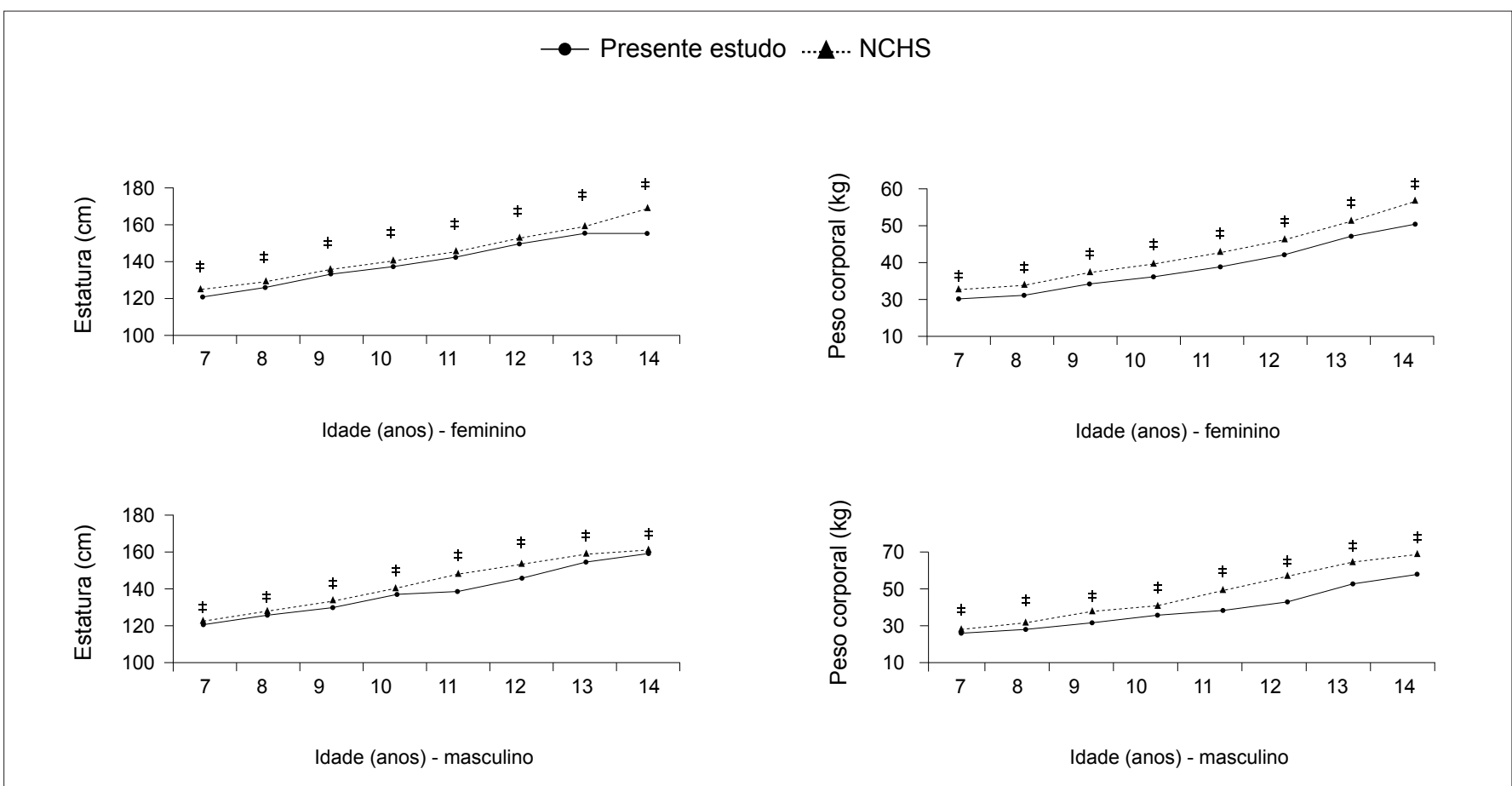

Figura 1 - Comparação da estatura e peso corporal das crianças e adolescentes do sexo masculino e feminino de Cotinguiba, Sergipe, com as curvas de referência do NCHS $\left({ }^{*} p<0,05\right)$.

Tabela 3 - Prevalência de desnutrição e excesso de peso corporal em crianças e adolescentes da região de Cotinguiba, Sergipe, segundo sexo e idade

\begin{tabular}{lcc}
\hline & Desnutrição & Excesso de peso \\
\cline { 2 - 3 } & $\mathrm{n}(\%)$ & $\mathrm{n}(\%)$ \\
\hline Total & $273(21,7)$ & $86(6,8)$ \\
Sexo & & \\
Masculino & $109(18,5)$ & $30(5,1)$ \\
Feminino & $164(24,6)$ & $56(8,4)$ \\
$p$ & 0,010 & 0,027 \\
Faixa etária (anos) & & \\
$7-9$ & $109(22,7)$ & $26(5,4)$ \\
$10-14$ & $164(21,1)$ & $60(7,7)$ \\
$p$ & 0,549 & 0,145 \\
\hline
\end{tabular}

Excesso de peso: sobrepeso+obesidade.

nos intervalos etários de 8-9, 11-12, 12-13 e 13-14 anos. Nos meninos, o aumento de peso foi de $5,8 \mathrm{~kg} / \mathrm{ano}$, com diferenças significantes nos intervalos 11-12 e 12-13 anos. Quanto ao IMC, no sexo feminino, foi verificado um aumento gradativo a partir dos 9-10 anos, com acréscimo médio mais elevado entre 13 e 14 anos $\left(1,30 \mathrm{~kg} / \mathrm{m}^{2}\right)$. No sexo masculino, o maior acréscimo médio ocorreu entre 11 e $12 \operatorname{anos}\left(1,29 \mathrm{~kg} / \mathrm{m}^{2}\right)$.

A Figura 1 apresenta a comparação da estatura e do peso corporal das crianças e adolescentes do presente estudo com as curvas de referência do $\mathrm{NCHS}^{(7)}$. Foram encontradas diferenças significativas em todas as idades e em ambos os sexos, sendo os valores assinalados nas curvas de referência estatisticamente superiores aos encontrados nos escolares avaliados $(p<0,05)$, com exceção das meninas, aos nove anos de idade.

Quanto ao estado nutricional (Tabela 3), os resultados demonstraram que a freqüência de desnutrição $(21,7 \%)$ foi aproximadamente três vezes mais elevada que a do excesso de peso $(6,8 \%)$. Na comparação entre os sexos, foi verificado que tanto a desnutrição quanto o excesso de peso foram mais prevalentes nas meninas do que nos meninos $(p<0,05)$. 


\section{Discussão}

Embora o presente estudo tenha utilizado dados de um levantamento de base escolar, é importante ressaltar que só foram incluídos os adolescentes que, na faixa etária estabelecida (7-14 anos), realizaram todas as medidas antropométricas (massa corporal e estatura). Apesar de a amostra não ter sido probabilística, acredita-se que os resultados aqui mostrados possam ser utilizados como um bom parâmetro de crescimento e estado nutricional dos escolares da rede estadual de ensino da região de Cotinguiba.

O maior crescimento estatural, no sexo feminino, ocorreu entre os 11 e 12 anos $(7,63 \mathrm{~cm} / \mathrm{ano})$ e, no masculino, entre os 12 e 13 anos ( $7,86 \mathrm{~cm} / \mathrm{ano})$, ou seja, o pico de crescimento de estatura nos meninos ocorreu cerca de dois anos mais tarde do que nas meninas, corroborando as evidências encontradas na literatura ${ }^{(18)}$. Em estudo realizado com adolescentes da região Sul do Brasil (Norte do Rio Grande do Sul e Oeste de Santa Catarina), verificou-se o pico de crescimento de estatura entre 12 e 14 anos no sexo masculino e, entre 11 e 13 anos, no feminino ${ }^{(5)}$. Em pesquisas conduzidas em diferentes locais do Brasil, os resultados revelam que o ganho médio anual de estatura varia de 5,0 a $5,6 \mathrm{~cm} /$ ano no sexo masculino e 4,4 a $5,0 \mathrm{~cm} /$ ano no feminino ${ }^{(19,20)}$.

Quanto ao peso corporal, os maiores incrementos médios foram encontrados, em ambos os sexos, entre 11 e 12 anos, com cerca de $6 \mathrm{~kg} / \mathrm{ano}$. Estudo realizado por Glaner ${ }^{(5)}$ mostrou maior ganho de peso corporal nos escolares do sexo feminino aos 13 anos e, no masculino, aos 15 e 17 anos. De acordo com a literatura nacional, o ganho médio anual em peso corporal de adolescentes varia de 3,3 a $4,7 \mathrm{~kg} / \mathrm{ano}$ no sexo masculino e de 2,7 a $4,5 \mathrm{~kg} /$ ano no feminino ${ }^{(19,20)}$.

Partindo do princípio de que as alterações no peso corporal, sobretudo em crianças e adolescentes, refletem as questões agudas referentes à nutrição, pode-se inferir que estas diferenciações ocorreram devido às influências ambientais, nutricionais ${ }^{(14)}$ ou maturacionais ${ }^{(18)}$, pois $\mathrm{o}$ incremento de peso está relacionado ao maior ganho de massa óssea e muscular nos meninos e ao maior ganho de tecido adiposo nas meninas ${ }^{(18)}$.

Quanto à comparação da estatura e peso corporal dos escolares do presente estudo com as curvas de referência do $\mathrm{NCHS}$, os resultados demonstraram valores superiores nas curvas do NCHS. Estes achados divergem dos encontrados em Ijuí, Rio Grande do Sul ${ }^{(11)}$, sendo os escolares de tal região mais altos e mais pesados do que a média da população analisada pelo NCHS.
Provavelmente devido às condições socioeconômicas inadequadas da região de Cotinguiba, verificou-se que a prevalência de desnutrição foi aproximadamente três vezes maior que a de excesso de peso. Tais resultados divergem dos achados de estudos de base populacional representativos da população brasileira, que indicam uma queda da desnutrição em crianças e adolescentes ${ }^{(21,22)}$. A desnutrição no Brasil parece se associar à região geográfica: nas regiões Norte e Nordeste, a desnutrição é pelo menos duas vezes maior do que na região Centro-Oeste e quatro vezes maior do que na região Sul ${ }^{(23)}$. Neste sentido, a desnutrição encontrada na região de Cotinguiba pode ser considerada um problema de Saúde Pública.

A prevalência de excesso de peso verificada nos escolares de Cotinguiba foi considerada baixa $(6,8 \%)$, sendo inferior à encontrada em pesquisas nacionais ${ }^{(4,24-26)} \mathrm{e}$ internacionais ${ }^{(26-28)}$.

Quanto ao sexo, observou-se que tanto a desnutrição quanto o excesso de peso foram mais freqüentes nas meninas (24,6 e 8,4\%, respectivamente) do que nos meninos (18,5 e $5,1 \%$, respectivamente). No que diz respeito à desnutrição, os resultados do presente estudo divergem dos encontrados na literatura ${ }^{(21,29)}$, que sugerem diminuição da desnutrição e aumento do excesso de peso. Da mesma forma, a prevalência de excesso de peso encontrada nos escolares da região de Cotinguiba é diferente dos resultados obtidos em Santos ${ }^{(30)}$, em São Paulo, e Florianópolis ${ }^{(31)}$, em Santa Catarina, onde os meninos apresentaram maior prevalência de excesso de peso do que as meninas. Em relação às pesquisas internacionais, os resultados também não são homogêneos. Na Itália, prevalências mais elevadas de excesso de peso são encontradas nos sexo masculino ${ }^{(32)}$ e, no México, no sexo feminino ${ }^{(33)}$.

Quando considerado o grupo etário dos escolares de Cotinguiba, tanto a prevalência de excesso de peso das crianças quanto a dos adolescentes foi menor que a observada em Salvador ${ }^{(34)}$, na Bahia, Recife ${ }^{(25)}$, em Pernambuco, e Pelotas ${ }^{(26)}$, no Rio Grande do Sul.

Esse estudo apresentou limitações inerentes ao seu desenho transversais. Nessa casuística, investigaram-se apenas escolares da rede estadual de ensino, impossibilitando verificar o crescimento físico e o estado nutricional de crianças e adolescentes da rede privada; não foram coletadas informações referentes ao nível socioeconômico das famílias dos escolares, contudo, indivíduos que freqüentam escolas da rede pública de ensino possivelmente pertencem a uma classe de menor renda em relação àqueles que freqüentam escolas privadas.

A partir dos resultados encontrados nos escolares de Cotinguiba, conclui-se que houve um pico de crescimento em 
estatura entre 11 e 13 anos para ambos os sexos, com maiores incrementos nos meninos quando comparados às meninas. Quanto ao peso corporal, os maiores incrementos foram observados entre 11 e 12 anos. Foi ainda verificada uma alta prevalência de desnutrição entre os escolares analisados, com maior proporção nas meninas do que nos meninos. Estes índices elevados de desnutrição em crianças e adolescentes da região de Cotinguiba sugerem a necessidade de acompanhamento longitudinal do crescimento e do estado nutricional, associado à implantação de programas nutricionais nas escolas. Nesta perspectiva, pode-se considerar que a avaliação nutricional de populações - a realização de inquéritos nutricionais no espaço escolar, em especial - é uma ferramenta de extrema importância para compreensão da dinâmica nutricional de crianças e adolescentes e, conseqüentemente, para a formulação de políticas de saúde e ações mais efetivas.

\section{Referências}

1. Rogol AD, Clark PA, Roemmich JN. Growth and pubertal development in children and adolescents: effects of diet and physical activity. Am J Clin Nutr 2000;72 (Suppl 2):521S-8.

2. Soares NT. Um novo referencial antropométrico de crescimento: significados e implicações. Rev Nutr 2003;16:93-104.

3. No authors listed. Use and interpretation of anthropometric indicators of nutritional status. WHO Working Group. Bull World Health Organ 1986;64:929-41.

4. Roman EP, Barros Filho AA. Differences on the growth and body composition between school children of German and Brazilian origin. Rev Paul Pediatr 2007;25:227-32.

5. Glaner MF. Physical growth in adolescents of the north gaúcho and west catarinense. Rev Bras Ci Mov 2005;13:15-20.

6. Hamill PV, Drizd TA, Johnson CL, Reed RB, Roche AF. NCHS growth curves for children birth-18 years. United States. Vital Health Stat 1977;11:1-74.

7. NCHS [homepage on the Internet]. 2000 CDC growth charts: United States [cited 2007 Jul 3]. Available from: http://www.cdc.gov/GROWTHCHARTS/

8. Victora CG, Araújo CL, de Onis M [homepage on the Internet]. Uma nova curva de crescimento para o século XXI. [cited 2008 Apr 4]. Available from: http:// dtr2004.saude.gov.br/nutricao/documentos/nova_curva_cresc_sec_xxi.pdf

9. Marques RM, Marcondes E, Bequó E, Hegg R, Colli AS, Zacchi MA. Crescimento e desenvolvimento pubertário em crianças e adolescentes brasileiros: II - altura e peso. São Paulo: Editora Brasileira de Ciências; 1982.

10. Brasil - Ministério da Saúde - Instituto Nacional de Alimentação e Nutrição. Perfil de crescimento da população brasileira de 0 a 25 anos: pesquisa nacional sobre saúde e nutrição. Brasília: Ministério da Saúde, 1990.

11. Diniz IMS, Lopes AS, Dummel CCB, Rieger T. Crescimento físico e adiposidade corporal de escolares. Rev Bras Cineantropom Desempenho Hum 2006;8:32-8.

12. Pires MC, Lopes AS. Crescimento físico e características sócio-demográficas em escolares no município Florianópolis - Sc, Brasil. Rev Bras Cineantropom Desempenho Hum 2004;6:17-26.

13. Barbetta PA. Estatística aplicada às ciências sociais. $2^{\mathrm{a}}$ ed. Florianópolis: UFSC; 1998.

14. World Health Organization. Physical status: the use and interpretation of anthropometry. Report of a WHO Expert Committee. World Heatlh Organ Tech Rep Ser 1995;854:1-452.

15. Alvarez BR, Pavan AL. Alturas e comprimentos. In: Petroski EL, editor. Antropometria: técnicas e padronizações. $2^{\mathrm{a}}$ ed. Porto Alegre: Pallotti; 2003. p. 29-51.

16. Cole TJ, Bellizzi MC, Flegal KM, Dietz WH. Establishing a standard definition for child overweight and obesity worldwide: international survey. BMJ 2000;320:1240-3.

17. Cole TJ, Flegal KM, Nicholls D, Jackson AA. Body mass index cut offs to define thinness in children and adolescents: international survey. BMJ 2007;335:194.

18. Malina RM, Bouchard C, Bar-Or O. Growth, maturation and physical activity. Champaign, Illinois: Human Kinetics Books; 1991.
19. Quadros TMB, Gordia AP, Pires Neto CS, Leite ML, Campos W, Kalinows FG Physical growth of school children of the private net of education of the city of Ponta Grossa, PR. Rev Bras Cineantropom Desempenho Hum 2006;8:3644.

20. Diniz IS, Lopes AS, Borgatto AF. Physical growth and body composition of students from distinct ethnic background of Rio Grande do Sul State, Brazil. Rev Bras Cineantropom Desempenho Hum 2008;10:12-8.

21. Anjos LA, Castro IR, Engstrom EM, Azevedo AM. Growth and nutritiona status in a probalistic sample of school children from Rio de Janeiro, 1999. Cad Saude Publica 2003;19 (Suppl 1):S171-9.

22. Monteiro CA, Conde WL. The secular tendency of obesity according to socia status: Northeast and Southeast of Brazil, 1975-1989-1997. Arq Bras Endocrinol Metab 1999;43:186-94.

23. Brasil - Instituto Brasileiro de Geografia e Estatística - Programa das Nações Unidas para o Desenvolvimento. Atlas do desenvolvimento humano no Brasil. Brasília: IBGE, 1997.

24. Giugliano R, Carneiro EC. Factors associated with obesity in school children J Pediatr (Rio J) 2004;80:17-22.

25. Silva GA, Balaban G, Motta ME. Prevalence of overweight and obesity in children and adolescents of different socioeconomic conditions. Rev Bras Saúde Mater Infant 2005;5:53-9.

26. Dutra CL, Araújo CL, Bertoldi AD. Prevalence of overweight in adolescents: a population-based study in a southern Brazilian city. Cad Saude Publica 2006;22:151-62.

27. Salazar-Martinez E, Allen B, Fernandez-Ortega C, Torres-Mejia G, Galal O, Lazcano-Ponce E. Overweight and Obesity status among adolescents from Mexico and Egypt. Arch Med Res 2006;37:535-42.

28. Moraes SA, Beltrán Rosas J, Mondini L, Freitas IC. Prevalence of overweight and obesity, and associated factors in school children from urban área in Chilpancingo, Guerrero, México, 2004. Cad Saúde Pública 2006;22:1289-301.

29. Souza OF, Cruz MD. Nutritional status of preschool children from Rio Branco/ AC. Rev Bras Cineantropom Desempenho Hum 2006;8:39-44.

30. Costa RF, Cintra IP, Fisberg M. Prevalência de sobrepeso e obesidade em escolares da cidade de Santos, SP. Arq Bras Endocrinol Metab 2006;50:60-7.

31. Farias Júnior JC, Lopes AS. Prevalence of overweight in adolescents. Rev Bras Ciên Mov 2003;11:77-84.

32. Celi F, Bini V, De Giorgi G, Molinari D, Faraoni F, Di Stefano G et al. Epidemiology of overweight and obesity among school children and adolescents in three provinces of central Italy, 1993-2001: study of potential influencing variables. Eur J Clin Nutr 2003;57:1045-51.

33. Hernández B, Cuevas-Nasu L, Shamah-Levy T, Monterrubio EA, Ramírez-Silva $\mathrm{Cl}$, García-Feregrino R et al. Factors associated with overweight and obesity in Mexican school-age children: Results from the National Nutrition Survey 1999. Salud Pública de Méx 2003;45 (Suppl 4):S551-7.

34. Leão LS, Araújo LM, Moraes LT, Assis AM. Prevalência de obesidade em escolares de Salvador, Bahia. Arq Bras Endocrinol Metab 2003;47:151-7. 